\title{
An 87-year-old man with a tympanic abdominal mass
}

A 87 -year-old man presented A with a 3-day history of progressive, diffuse abdominal pain. He denied nausea, vomiting, fever or chills. He had no history of rectal bleeding, diarrhea or constipation, but he had noticed increased flatulence, which helped relieve the pain. His vital signs were normal. He had a distended, diffusely tender abdomen with a large left upper quadrant mass that was tympanic to percussion. A digital rectal examination was normal, but his stool was positive for occult blood. His leukocyte count was normal, but his hemoglobin level was low at 82 (normally 110-150) g/L, \& which was consistent with a 2year history of anemia, for which the patient had refused investigation. Plain radiographs demonstrated a large radiolucent structure $(11 \mathrm{~cm} \times 22 \mathrm{~cm})$ in the upper abdomen with no signs of obstruction (Fig. 1).
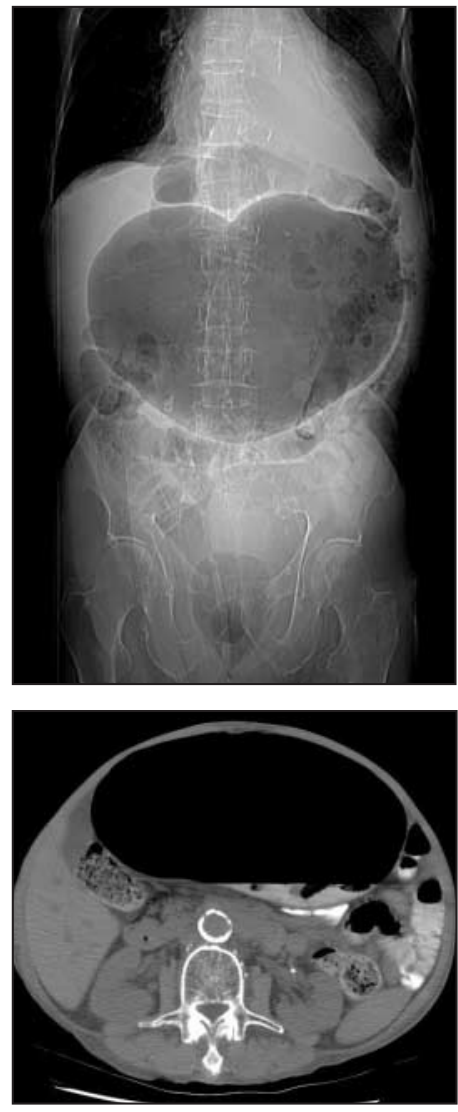

The patient was admitted to hospital for further investigation and was managed conservatively with bowel rest, intravenous fluids, pain management and antibiotics for suspicion of diverticulitis. A CT scan revealed that the structure was continuous with the sigmoid colon (Fig. 2), which was confirmed when contrast material administered through the rectum entered the smooth-walled structure. The mass was diagnosed as a giant sigmoid diverticulum. The patient's pain ceased after the passage of a large amount of flatus the next day. After no further rectal bleeding the patient was discharged home, having refused further management.

Giant colonic diverticulum is rare, with fewer than 100 cases reported in the literature. It affects men and women equally, occurs most commonly in patients $60-80$ years of age and affects the sigmoid colon in over $90 \%$ of cases. ${ }^{1}$ Patients may be asymptomatic or have nonspecific symptoms, such as fever, nausea, vomiting, diarrhea, constipation, or abdominal pain or distension, or they may present with complications such as perforation (followed by pneumoperitoneum), diverticulitis, abscess formation, gastrointestinal hemorrhage or bowel obstruction. ${ }^{2,3}$ In addition, giant diverticula carry a $2 \%$ risk of adenocarcinoma of the involved colon at the time of presentation. ${ }^{3}$

The mechanism by which ordinary diverticula become giant diverticula is not fully understood. One theory is that colonic inflammation narrows the outlet and creates a "ball-valve" mechanism that allows air to enter but not escape the diverticulum, which results in its progressive dilatation. Other theories sug- gest that gas-producing microorganisms cause the diverticulum expansion, or that a small colonic perforation causes pseudocyst formation, followed by the "ball-valve" mechanism. ${ }^{1,2}$

Abdominal radiographs will show a large round or elliptical lucency with smooth margins ${ }^{2}$ that may contain an air-fluid level. ${ }^{1} \mathrm{~A}$ barium enema results in the opacification of $60 \%$ of diverticula, and if the contrast penetrates into the diverticulum then the borders should be smooth. Irregular borders should raise the suspicion of chronic inflammatory or neoplastic changes. ${ }^{4} \mathrm{CT}$ scans will show a large gas-filled cavity with smooth margins that is continuous with the colon.

Treatment includes conservative management such as pain control and colonic decompression as needed. Surgical intervention such as diverticulectomy or colectomy may be required if abdominal pain becomes intractable or profuse bleeding occurs. Because of the risk of recurrence and adenocarcinoma, surgical resection is felt by some to be the preferred management in all cases. ${ }^{3}$

\section{Dmitry Guller}

Faculty of Medicine and Dentistry David Bach

Department of Diagnostic Radiology and Nuclear Medicine

University of Western Ontario

London, Ont.

\section{References}

1. Havenstrite KA, Harris JA, Rivera DE. Giant colonic diverticulum: report of a case. Am Surg 1999;65(6):578-80.

2. Roger T, Rommens J, Bailly J, Vollont GH, Belva P, Delcour C. Giant colonic diverticulum: presentation of one case and review of the literature. Abdom Imaging 1996;21:530-3.

3. Steenvoorde P, Vogelaar FJ, Oskam J, Tollenaar RAEM. Giant colonic diverticula. Dig Surg 2004;21:1-6.

4. Moesgaard J, Felding C. Giant diverticulum of the sigmoid colon. Acta Chir Scand 1983;149:445-7.

5. Fields S, Haskell L, Libson E. CT appearance of giant colonic diverticulum. Gastrointest Radiol 1987;12:73-5. 